Background It is estimated that everyday over 1 million individuals contract a curable sexually transmitted infection (STI) worldwide. For an appropriate STI treatment, it is necessary to have an accurate diagnosis. The Alinity $\mathrm{m}$ STI assay is a multiplex RT-PCR assay that identifies four STI pathogens: Chlamydia trachomatis (CT), Neisseria gonorrhoeae (NG), Trichomonas vaginalis (TV), and Mycoplasma genitalium (MG) in a single $(115 \mathrm{~min})$ reaction. The aim of this study was to evaluate the assay clinical performance.

Methods Clinical performance of Alinity m STI assay was assessed using 201 residual clinical samples [119 urine and 82 in gynecological specimens] and compared with Abbott RealTime CT/NG assay and XGEN MULTI UP test (Mobius Life Science) for TV/MG. Precision and reproducibility were evaluated by testing panel members in contrived swab. Five panel members (PM) were tested in 12 replicates in two days: $\mathrm{PM} 1=\mathrm{CT}, \mathrm{PM} 2=\mathrm{NG}, \mathrm{PM} 3=\mathrm{TV}, \mathrm{PM} 4=\mathrm{MG}$ and $\mathrm{PM} 5=\mathrm{CT} /$ $\mathrm{NG} / \mathrm{TV} / \mathrm{MG}$ at $2 \mathrm{X}$ claimed LoD.

Results For CT, the positive (PPA) agreement and negative (NPA) agreements were $95 \%$ and $100 \%$ respectively. For NG, the PPA was $94 \%$ for urine and $100 \%$ for gynecological specimens, and NPA was 99\% and 100\%, respectively. For MG, PPA and NPA were 100\%. For TV, NPA was 100\% (no positive result obtained). Co-infection with $\mathrm{MG}$ was observed in $5 \%$ of CT or NG positive samples. The overall agreement for both sample types and the four organisms was 98.5\% (516/ 524). All panel members were detected and accurately identified, individually (PM1-4) or in the presence of the other three pathogens (PM5).

Conclusion The Alinity m STI assay showed excellent agreement (97-100\%) between methods and streamlines laboratory workflow with simultaneous detection of 4 pathogens in a single reaction from the same sample. This assay allows rapid infection identification supporting clinicians to properly treat patients, especially when a co-infection is present.

\section{P270 ACCEPTABILITY OF SELF-COLLECTED THROAT SWABS AMONG MEN WHO HAVE SEX WITH MEN ATTENDING A SEXUAL HEALTH CENTRE}

${ }^{1,2} \mathrm{~T}$ Phillips*, ${ }^{1,2} \mathrm{C}$ Fairley, ${ }^{1,2} \mathrm{C}$ Bradshaw, ${ }^{2} \mathrm{~K}$ Maddaford, ${ }^{2} \mathrm{E}$ Rodriguez, ${ }^{2} \mathrm{~S}$ Hall, ${ }^{1,2,3} \mathrm{E}$ Chow. ${ }^{1}$ Monash University, Clayton, Australia; ${ }^{2}$ Melbourne Sexual Health Centre, Carlton, Australia; ${ }^{3}$ The University of Melbourne, Parkville, Australia

\subsection{6/sextrans-2021-sti.341}

Background Due to the COVID-19 pandemic, sexual health clinics across Australia have switched from clinician-collected to self-collected swabs for oropharyngeal STI screening. The study aimed to determine the acceptability of self-collected throat swabs among 200 gay, bisexual and other men who have sex with men (GBMSM) attending a sexual health service.

Methods GBMSM aged $\geq 16$ years old, had a throat swab taken at the Melbourne Sexual Health Centre (MSHC), and provided consent to participate in research, were invited to take this online survey by SMS. The survey collected data on how easy or difficult men found collecting their own throat swab.

Results 273 GBMSM completed the survey; 218 (79.9\%) selfcollected their throat swab and were included in the final analysis. Most participants found collecting their own throat swabs very easy, easy, or were neutral $(190 ; 87.2 \%$; 95\% confidence interval $[95 \% \mathrm{CI}]: 82.0 \%$ to $91.3 \%)$ and $28(12.8 \%$; 95\% CI: $8.7 \%$ to $18.0 \%$ ) found it difficult or very difficult. There were 85 (39.0\%) who reported taking the swab by themselves was worse than previous experiences of cliniciancollected swabs, compared to 110 (50.5\%) who reported it was better taking the swab by themselves or found no difference to previous clinician-collected swabs. The two most commonly reported experiences during self-collection were gagging $(155 ; 71.1 \%)$ and being worried they had not done it correctly (46 out of 95 who were asked this; 48.4\%). Almost half of participants preferred to have a clinician take their throat swab (103; 47.5\%; 95\%CI: $40.5 \%$ to $54.1 \%) ; 66$ (30.4\%; CI: $24.3 \%$ to $36.8 \%)$ did not have a preference and $48(22.1 \%$; $95 \% \mathrm{CI}: 16.7 \%$ to $28.1 \%)$ preferred to take the swab themselves.

Conclusion Most GBMSM did not find self-collecting throat swabs difficult, however almost half of participants preferred to have a clinician take the swab.

\section{P271 SEXUAL HEALTH SERVICE ADAPTATIONS TO THE CORONAVIRUS DISEASE 2019 (COVID-19) PANDEMIC IN AUSTRALIA: A NATIONWIDE ONLINE SURVEY}

\begin{abstract}
${ }^{1,2}$ T Phillips $^{*},{ }^{1,2} \mathrm{C}$ Fairley, ${ }^{3,4} \mathrm{~B}$ Donovan, ${ }^{1,2}$ J Ong, ${ }^{4} \mathrm{~A}$ McNulty, ${ }^{5} \mathrm{~L}$ Marshall, ${ }^{3,6} \mathrm{D}$ Templeton, ${ }^{7} \mathrm{~L}$ Owen, ${ }^{8} \mathrm{~A}$ Ward, ${ }^{9} \mathrm{M}$ Gunathilake, ${ }^{10} \mathrm{D}$ Russell, ${ }^{11} \mathrm{~J}$ Langton-Lockton, ${ }^{3,4,12} \mathrm{C}$ Bourne, ${ }^{13} \mathrm{~S}$ Martin, ${ }^{1,2,14} \mathrm{E}$ Chow. ${ }^{1}$ Monash University, Clayton, Australia; ${ }^{2}$ Melbourne Sexual Health Centre, Carlton, Australia; ${ }^{3}$ Kirby Institute, UNSW Sydney, Sydney, Australia; ${ }^{4}$ Sydney Sexual Health Centre, Sydney, Australia; ${ }^{5}$ Fremantle Hospital and Health Service, Fremantle, Australia; ${ }^{6}$ Department of Sexual Health Medicine, Sydney Local Health District, Camperdown, Australia; ${ }^{7}$ Statewide Sexual Health Services, Australia; ${ }^{8}$ Adelaide Sexual Health Centre, Adelaide, Australia; ${ }^{9}$ Sexual Health and Blood Borne Virus Unit of Centre for Disease Control, Australia; ${ }^{10}$ Cairns Sexual Health Service, Cairns North, Australia; ${ }^{11}$ Queensland Health, Brisbane, Australia; ${ }^{12}$ Centre for Population Health, Australia;

${ }^{13}$ Canberra Hospital, Canberra, Australia; ${ }^{14}$ The University of Melbourne, Parkville, Australia
\end{abstract}

\subsection{6/sextrans-2021-sti.342}

Background We aimed to examine the changes public sexual health services across Australia made during the national lockdown (March-May 2020) due to the COVID-19 pandemic.

Methods From July-August 2020, we emailed a link to an online survey to 21 sexual health clinic directors/managers who were part of the Australian Collaboration for Coordinated Enhanced Sentinel Surveillance of Sexually Transmissible Infections and Blood-borne Viruses (ACCESS) network.

Results All 20 participating clinics remained open but reported changes during the lockdown, including suspension of walk-in services in 8 clinics.

Some clinics stopped offering asymptomatic screening for heterosexuals $(n=11)$, men who have sex with men (MSM) $(n=3)$, or transgender persons $(n=2)$. Most clinics offered a mix of telehealth and face-to-face consultations for asymptomatic MSM $(\mathrm{n}=11)$, asymptomatic transgender persons $(n=12)$, post-exposure prophylaxis (PEP) prescription $(n=13)$ or to initiate pre-exposure prophylaxis (PrEP) $(n=14)$. People who were symptomatic for STIs and contacts of STIs were offered face-to-face and telehealth consultations across all clinics. Seven clinics suspended STI test-of-cure consultations and four clinics suspended hepatitis vaccinations for people not living with HIV. Nineteen clinics reported delays in testing and 13 reported limitations in testing during lockdown. Most clinics changed to phone consultations for HIV 